\title{
Gender Differences in Familial Status, Socioeconomics, Functional Capacities and Wellbeing Among Oldest-Old Aged 80 Years and Above - China, 1998-2018
}

\author{
Yi Zeng ${ }^{1,2, *}$
}

\section{Summary \\ What is already known on this topic? \\ Little is known about gender differences in health, family, and socioeconomics among oldest-old in China, What is added by this report? \\ This study aims to fill this knowledge gap through analyzing gender differences in familial status, socioeconomics, functional capacities, and self-reported wellbeing using the 1998, 2008-2009, and 2017-2018 waves of the Chinese Longitudinal Healthy Longevity Survey. We found that compared with the male oldest- old, the female oldest-old are seriously disadvantaged in familial status, socioeconomics, and capacities of activities of daily living, physical performances, cognitive function, and self-reported wellbeing. \\ What are the implications for public health practice? \\ We discussed possible contributing reasons for our findings and strongly suggest that the female oldest-old should be prioritized higher.}

The numbers of oldest-old aged 80 years and above who will most likely need care in daily life and health services will climb from nearly 20 million in 2010 to about 120 million in 2050 in China (1). However, little is known about the oldest-old in most developing countries including China (Supplementary Materials, available in http://weekly.chinacdc.cn/). To fill in this knowledge gap for scientific studies and policymaking, this paper presents easily understandable analyses of gender differentials in familial status, socioeconomics, activities of daily living (ADL), physical performance, cognitive functions, self-reported health, and life satisfaction among the oldest-old aged 80 years and above in China. The definitions of the measurements of the activities of daily living, physical performance, self-reported health, and life satisfaction are described in "measurements of the health statuses" of Supplementary Materials.

The Chinese Longitudinal Healthy Longevity Study
(CLHLS) was conducted in 1998-2018 and purposefully over-sampled the oldest-old aged 80 years and above, plus a compatible sub-sample of youngerold aged 60-79. We use the datasets of oldest-old aged 80 years and above collected in 1998, 2008-2009, and 2017-2018 waves of CLHLS in the present study to perform meaningful comparative analyses on gender differences in the 3 periods (each roughly 10 years apart) from 1998 to 2018 . The included sample sizes were 8,805 oldest-old in the 1998 wave, 12,281 oldestold in the 2008-2009 wave, and 10,437 oldest-old in the 2017-2018 wave. $P$ values vary among different 5 year-old age groups and periods. In total, there are 210 $P$ values in Figures 1-4 that are available upon request to the author.

As shown in Figure 1A, the proportion of female oldest-old who were married was lower than their male counterparts. However, the overwhelming majority were widowed, especially the oldest-old women: $66 \%-98 \%$ of female oldest-old were widows, which was much higher than male oldest-old. The proportions of never-married and divorced male and female oldest-old were extremely low.

Figure 1B presented sex-age-specific percentage distributions of living with spouse and/or children (including children, grandchildren, and other family members), and those living alone. The overwhelming majority of Chinese oldest-old lived with spouses and/or children: $68 \%-90 \%$ for females, $80 \%-89 \%$ for males. Female oldest-old were less likely live with spouse/children up to ages 90-94, and the proportion of oldest-old women who lived alone were higher than the oldest-old men up to ages 90-94.

Figure $2 \mathrm{~A}$ indicated that about $66 \%-93 \%$ of female oldest-old were illiterate with no schooling, in contrast to $29 \%-58 \%$ of their male counterparts. Many Chinese oldest-old women were found to be illiterate, and females were significantly disadvantaged.

Figure $2 \mathrm{~B}$ indicated that percent of pension receivers among Chinese female oldest-old were much lower than their male counterparts. The majority of the 
(A1) 1998 wave

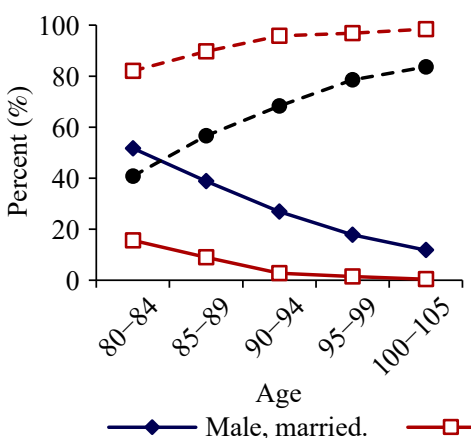

(B1) 1998 wave

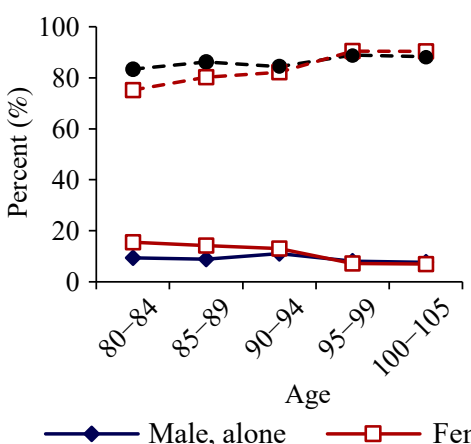

(A2) 2008-2009 wave

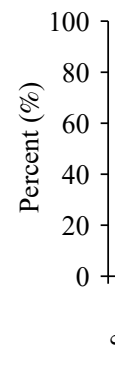

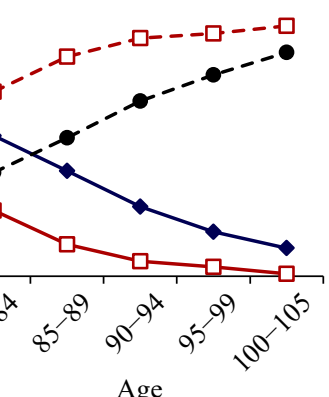

Age

Female, married. - - - - Male, widowhood.

(B2) 2008-2009 wave

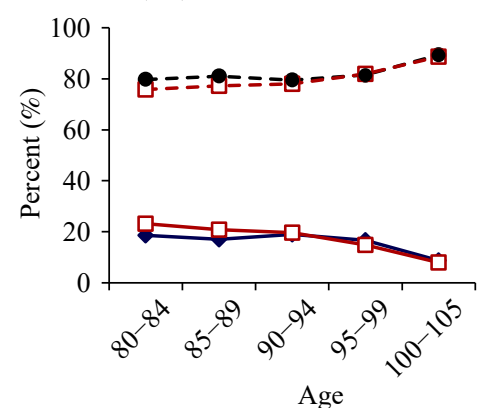

Age

- - - - Male, with spouse/child

(A3) 2017-2018 wave

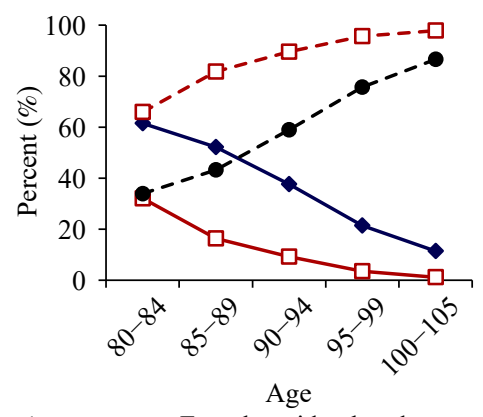

- - - - - Female, widowhood.

(B3) 2017-2018 wave

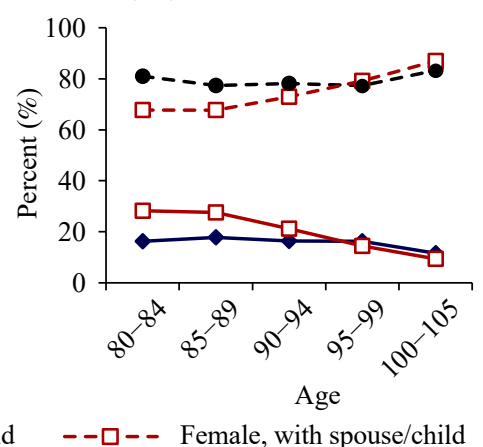

FIGURE 1. Gender differences in familial status among the oldest-old in China, 1998-2018. (A) Percentage of marital statuses among the oldest-old; (B) Percentage of living arrangements among the oldest-old.

Chinese oldest-old rely mainly on their children for financial support, and the gender differentials in primary source of financial supports were enormous: oldest-old women rely on their children much more than oldest-old men (Figure 2C).

About $48 \%-89 \%$ of oldest-old men's primary caregivers were their children, in contrast to $75 \%-92 \%$ of their female counterparts (Figure 2D). The female oldest-old in China relied more on their children as caregivers, as compared to male oldest-old whose primary caregivers were more likely their spouse. This was consistent with a much higher proportion of widowhood existing in female oldest-old than in male oldest-old. Children were often the primary resource of financial support of the oldest-old and also the primary resource of caregiving when the oldest-old were sick.

As shown in Figure 3A, percent of activities of ADL active status declined quickly after age 80 , especially after age 85-89. The curve of females who were ADL active was substantially lower than the male curve after age $80-84$. The oldest-old women were substantially less active than the oldest-old men in daily living.

Figure $3 \mathrm{~B}$ indicated that about $80 \%-88 \%$ and $77 \%-85 \%$ of male and female oldest-old aged $80-85$, respectively, could stand-up from a chair without using their hands; but the percent decreased quickly after age 85-89. About 38\%-53\% male centenarians could stand-up from a chair without using their hands, compared to $27 \%-37 \%$ female centenarians. Female oldest-old persons' capacities to perform picking-up a book on the floor from standing position were significantly worse than their male counterparts (Figure 3C). Male oldest-old physically performed better than female oldest-old did, and the gender gap became larger after age 90-94.

Figure 3D demonstrates that percent of good cognitive function among the Chinese oldest-old declined quickly with increase of age. The Chinese female oldest-old persons' cognitive capacity was much worse than their male counterparts, and the gender gaps were remarkably large.

As shown in Figure 4A, about $40 \%-62 \%$ of the male oldest-old reported "good health" in contrast to the corresponding percentages of $34 \%-59 \%$ of the female oldest-old. The percentages of self-reported good health among Chinese oldest-old declined slightly from age $80-84$ to age $100-105$, and even slightly increased from ages $95-99$ to ages $100-105$ in 2008-2009 and 2017-2018, respectively among females at these very advanced ages. Such pattern of age variations differed from the age patterns of $\mathrm{ADL}$, 
(A1) 1998 wave

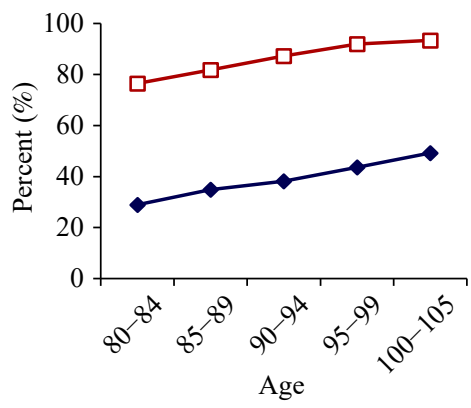

(B1) 1998 wave

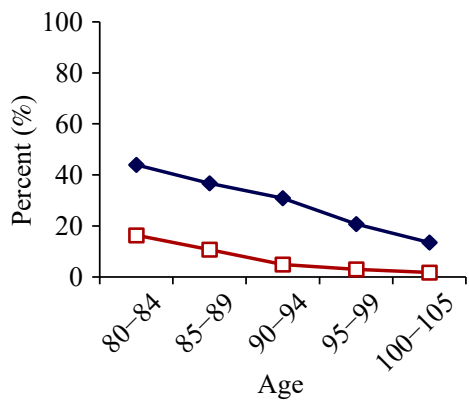

(C1) 1998 wave

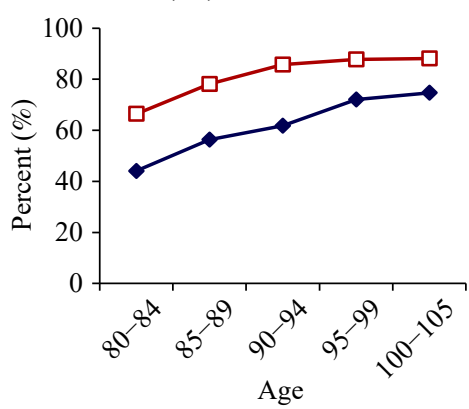

(D1) 1998 wave

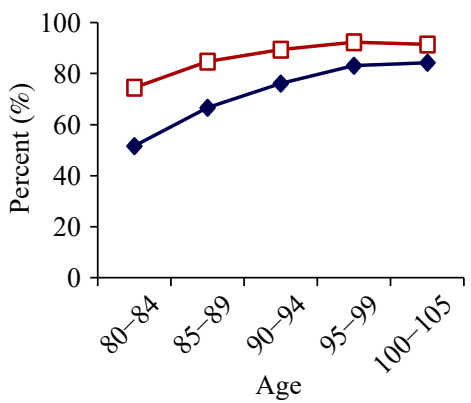

(A2) 2008-2009 wave

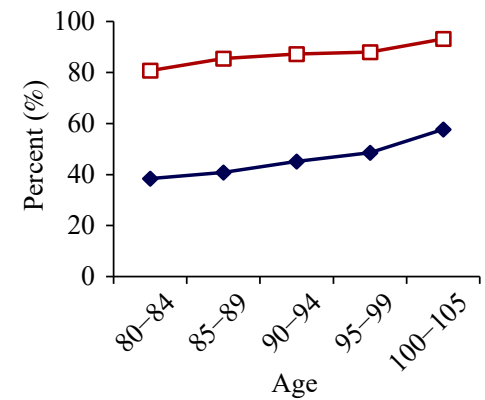

(B2) 2008-2009 wave

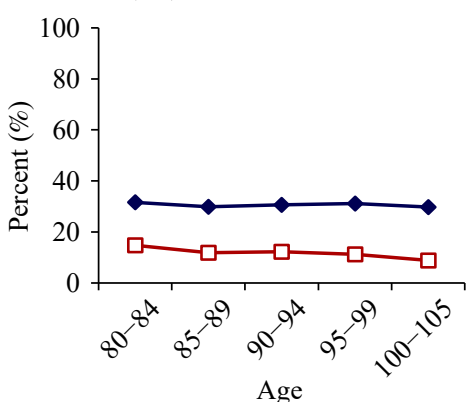

(C2) 2008-2009 wave

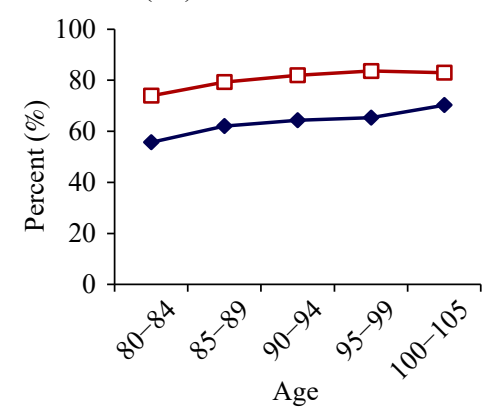

(D2) 2008-2009 wave

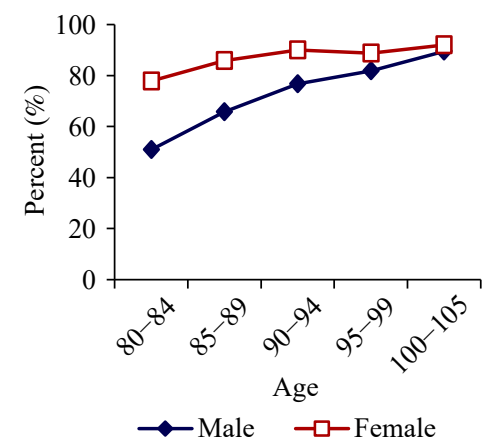

(A3) 2017-2018 wave

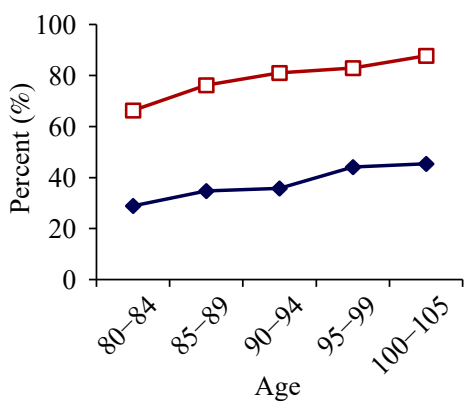

(B3) 2017-2018 wave

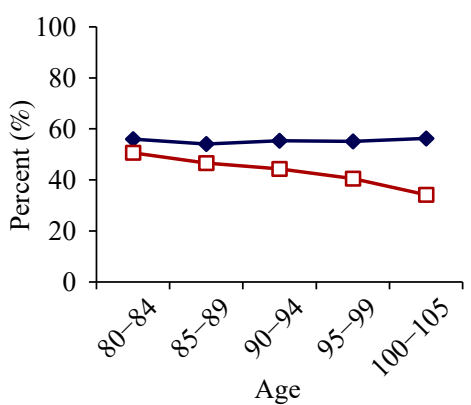

(C3) 2017-2018 wave

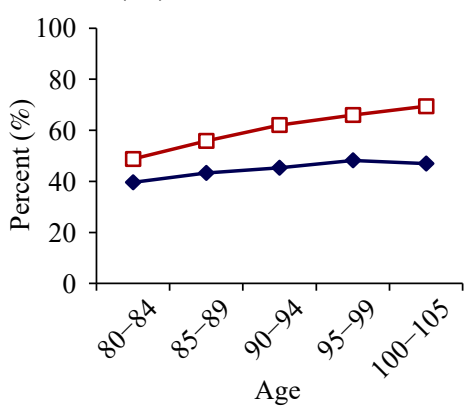

(D3) 2017-2018 wave

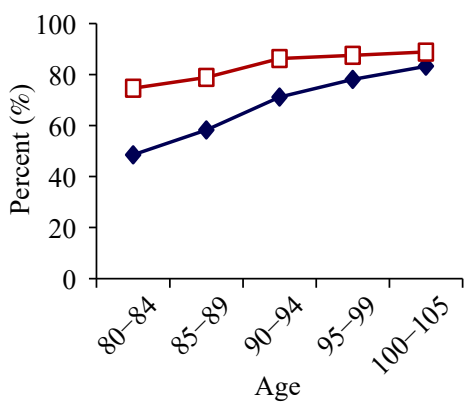

FIGURE 2. Gender differences in socioeconomic statuses among the oldest-old in China, 1998-2018. (A) Percent of illiterate among the oldest-old. (B) Percent of pension receivers among the oldest-old. (C) Percent of the oldest-old who mainly rely on children for financial support. (D) Percent of the oldest-old whose primary caregivers were children.

physical performance, and mini-mental state examination (MMSE). Figure 4D presented the results of self-reported good life satisfaction, which showed that gender differences in self-reporting good life satisfaction among Chinese oldest-old were mostly negligible in 1998, 2008-2009, and 2017-2018.

\section{DISCUSSION}

Populations of the oldest-old aged 80 years and over are growing extremely quickly in China and worldwide. Figures 1-4 demonstrated that female oldest-old were disadvantaged as compared to their 
(A1) 1998 wave

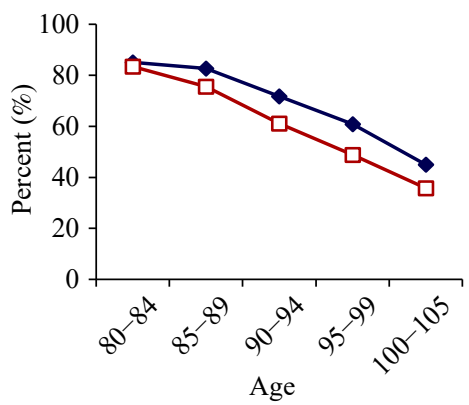

(B1) 1998 wave

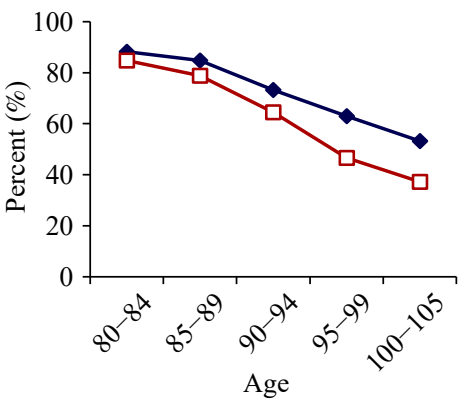

(C1) 1998 wave

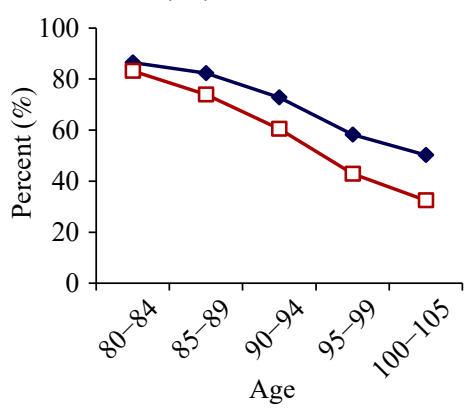

(D1) 1998 wave

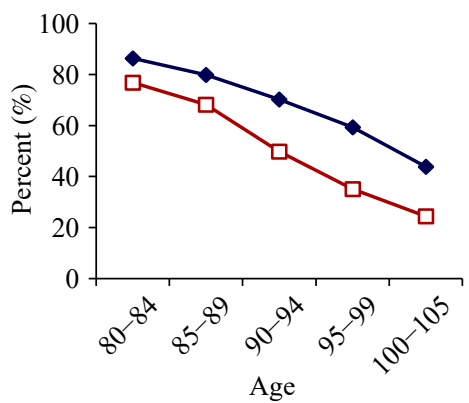

(A2) 2008-2009 wave

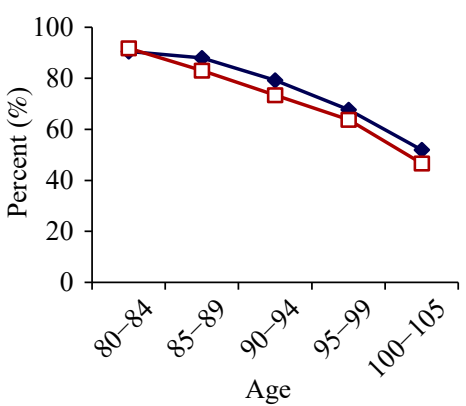

(B2) 2008-2009 wave

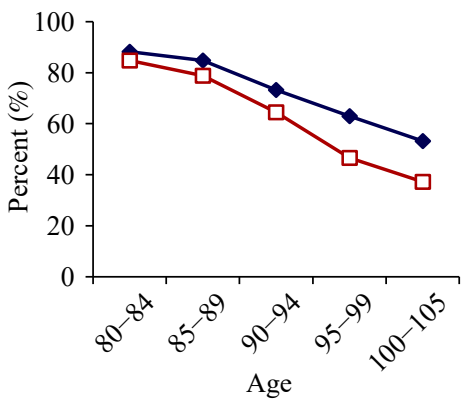

(C2) 2008-2009 wave

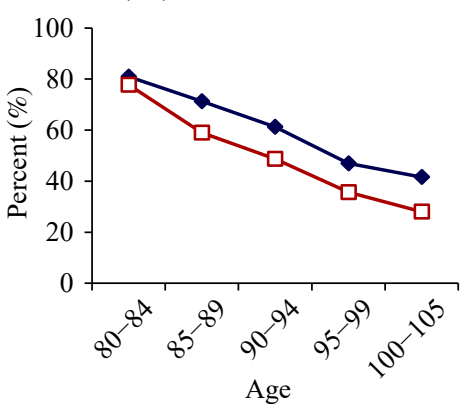

(D2) 2008-2009 wave

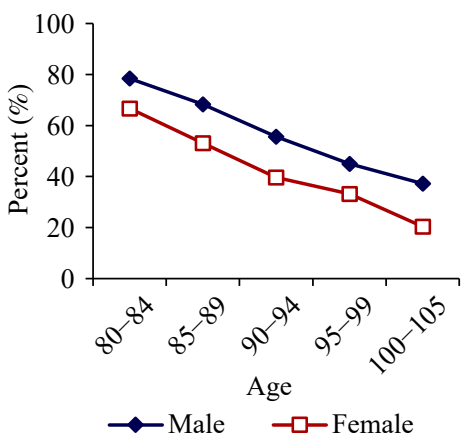

(A3) 2017-2018 wave

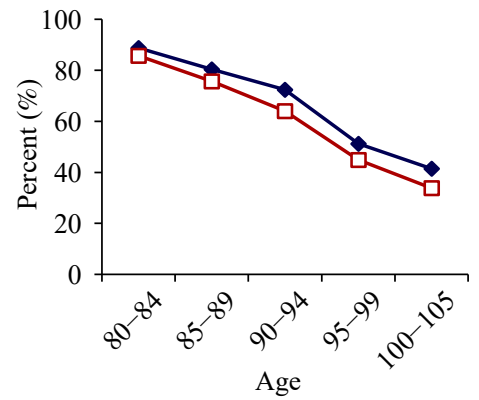

(B3) 2017-2018 wave

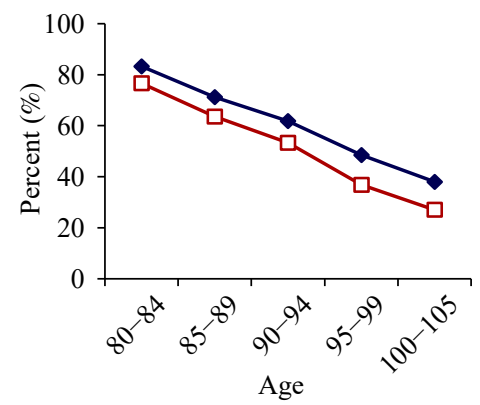

(C3) 2017-2018 wave

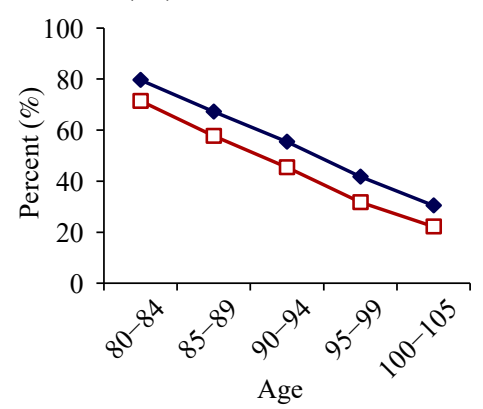

(D3) 2017-2018 wave

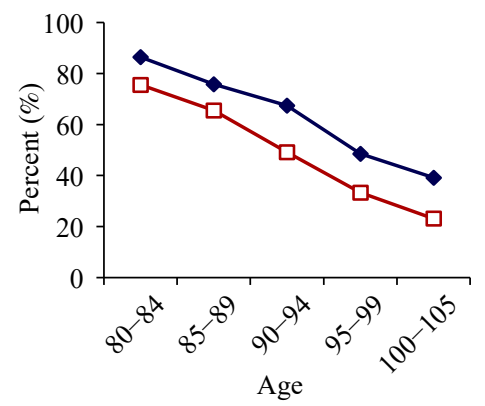

FIGURE 3. Gender differences in functional capacities among the oldest-old in China, 1998-2018. (A) Percent of the oldestold who were active in activities of daily living (ADL). (B) Percent of the oldest-old who could stand-up from a chair without using their hands. (C) Percent of the oldest-old who could pick-up a book on the floor from standing position. (D) Percent of the oldest-old whose cognitive functional capacity is good.

male counterparts. Oldest-old women were much more likely to live alone as they have higher risk of losing husbands due to higher mortality rates in male oldestold, while female widows have lower likelihood to remarry. Compared to male oldest-old, the proportion of pension receivers among female oldest-old was much lower, and they were often less educated, economically more dependent, and more reliant on children for financial support and for care when sick.

Oldest-old women were more likely to be disabled in activities of daily living, physical performances, and cognitive function. Other studies in China (2-3) and 
(A1) 1998 wave

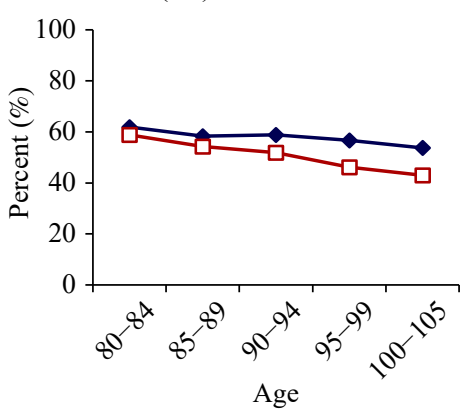

(B1) 1998 wave

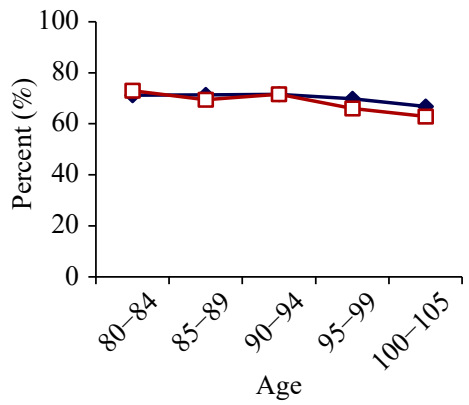

(A2) 2008-2009 wave

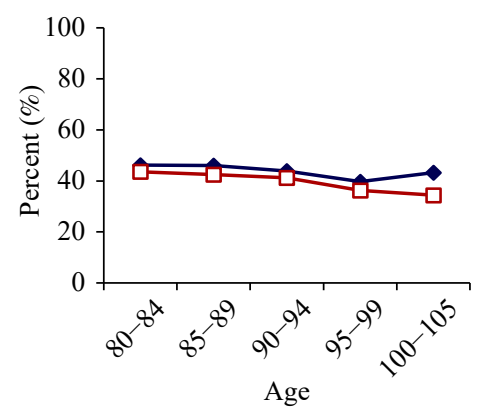

(B2) 2008-2009 wave

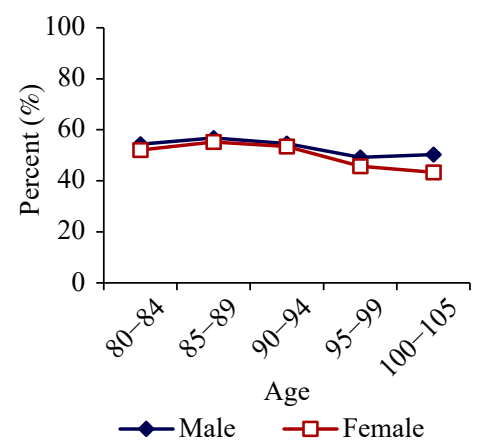

(A3) 2017-2018 wave

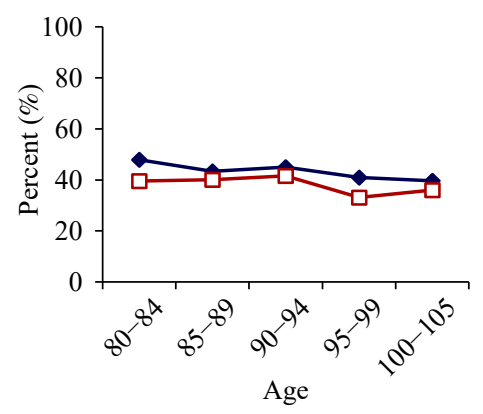

(B3) 2017-2018 wave

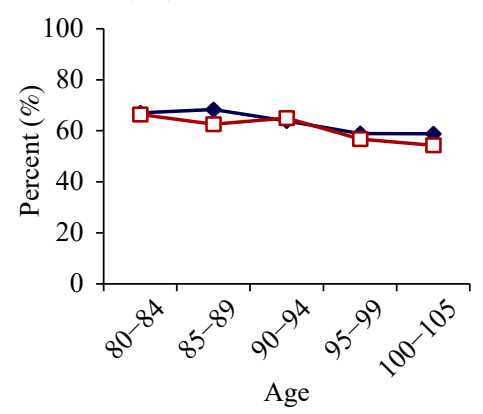

FIGURE 4. Gender differences in self-reported health and life satisfaction among the oldest-old in China, 1998-2018. (A) Percent of the oldest-old who self-reported "good health". (B) Percent of the oldest-old who self-reported "good life satisfaction".

elsewhere (4-5) also reported that elderly women were more disabled than men. Self-reported good health did not decline significantly with increase in age, which may be due to declining expectations of health with increasing age among the oldest-old in China and elsewhere (G). Furthermore, gender differences in selfreported life satisfaction among the Chinese oldest-old were mostly negligible as demonstrated in all of the datasets of CLHLS 1998, 2008-2009, and 2017-2018 waves (Figure 4B).

At the same time, however, the oldest-old Chinese women were seriously disadvantaged in marital status, living arrangements, education, pensions, financial resource, $\mathrm{ADL}$, physical performance, and cognitive function as compared to their male counterparts (Figures 1-3). Female oldest-old were also disadvantaged (to a less serious degree) in self-reported health, as shown in Figure 4. Chinese oldest-old women may be more positive in facing life's challenges and their general expectations in life satisfaction may be lower than their male counterparts. The percentages of self-reported good health and life satisfaction among Chinese male and female oldest-old did not decline or declined only slightly across age groups (Figure 4), while their functional capacities declined quickly with increases in age (Figure 3).

Female oldest-old's life expectancy at ages 80 and 90 were $18.7 \%$ and $16.0 \%$ higher, respectively, than that of male oldest-old in China in 2020 (article in submission; email Corresponding Author for more information). These contributed to the well-known "male-female health-survival paradox" (7). Similar gender differentials have also been found in other countries. For example, based on the data from the U.S. Long Term Care Surveys (1982-1994), Manton and Land (8) found that, although American oldest-old women have a longer total life expectancy at the age of 80 and 85 , they have significantly shorter active life expectancy as compared to American oldest-old men; such female disadvantages continue to the end of the U.S. health life tables.

Three preliminary explanations may shed light on understanding the "male-female health-survival paradox" and the gender differentials of the familial, socioeconomic and health statuses in China and elsewhere. First, the lifestyle of smoking and drinking strong alcohol was much lower among female oldestold than male oldest-old in China, which contributed to oldest-old women's longer lifespan. It was generally considered inappropriate for a woman to smoke or to attend a formal dinner (or banquet) at which the male hosts and participants drank a lot of strong alcohol in the traditional Chinese society. Thus, female Chinese oldest-old were subjected to a substantially lower risk 
of smoking and drinking strong alcohol that reduced lifespan.

Second, as compared to their male counterparts, female oldest-old were less likely to perform outdoor physical activities, such as farming, exercising, and fishing. Oldest-old women were also less likely engaged in reading, writing, meetings, and social and cultural activities, as they have lower levels of education and socioeconomic status. Female oldest-old persons' lower frequency of performing outdoor activities and lower education levels and socioeconomic status gave them fewer opportunities to maintain their capacities in activities of daily living, physical performance, cognitive function, and to obtain health services when they were sick.

Third, we recently discovered that genetic associations with longevity benefit females significantly more than males through analyzing datasets of genome-wide association study on 2,178 centenarians and 2,299 middle-age controls of CLHLS participants. This novel discovery was replicated across North and South regions of China and was further reconfirmed by analyses of different datasets of Chinese healthy aging candidate genes with 2,972 centenarians and 1,992 middle-age controls of CLHLS participants (article in submission; email Corresponding Author for more information).

In conclusion, the CLHLS 20-years longitudinal survey observations have consistently demonstrated the oldest-old women's serious disadvantages in familial and socioeconomic statuses, capacities of activities of daily living, physical performance and cognitive function, and self-reported health and life satisfaction. These findings imply that long-term care service programs should take into account the disadvantaged status of the female oldest-old. Government at all levels and society more broadly need to pay attention to enormous gender differentials in pensions and main source of financial support for the oldest-old persons. Very careful attention in policymaking and implementation should be given to ensure that any old age insurance and social service programs be developed or reformed to better prioritize the female oldest-old.

While the present study made significant contributions in providing empirical evidences of gender differences in familial, socioeconomic and health statuses of oldest-old aged 80 years and over in China, it also had limitations. In this paper, we adjusted only for genders and ages to ascertain the factual gender differentials in marital status, living arrangements, socioeconomic status, capacities in activities of daily living, physical performance, cognitive functions, self-reported health, and life satisfaction. However, other possible confounding factors were not controlled for. For example, as demonstrated in Figure 3D, we discovered that the gender differences in cognitive function among the Chinese oldest-old were extremely large. Due to space limits and the policy analyses nature of the present paper, we did not quantitatively explore which of the complicated factors caused such a large gender gap. We will conduct in-depth investigations on the causal determinants and impact factors of the gender differences in our future academic research articles, through employing the multivariate statistical methods such as sophisticated regression analyses.

Funding: National Key R\&D Program of China (2018YFC2000400), National Natural Sciences Foundation of China (72061137004, 71490732), the U.S. NIA/NIH (P01AG031719) and Duke/Duke NUS/RECA (Pilot)/2019/0051.

doi: $10.46234 / \mathrm{ccdcw} 2021.188$

\# Corresponding author: Yi Zeng, zengyi@nsd.pku.edu.cn.

${ }^{1}$ National School of Development, Center for Healthy Aging and Development Studies, Peking University, Beijing, China; ${ }^{2}$ Center for Study of Aging and Human Development and Geriatrics Division, School of Medicine, Duke University, Durham, NC, USA.

Submitted: June 23, 2021; Accepted: August 24, 2021

\section{REFERENCES}

1. Zeng Y, Wang ZL. A policy analysis on challenges and opportunities of population/household aging in China. J Popul Ageing 2014;7(4):255 81. http://dx.doi.org/10.1007/s12062-014-9102-y.

2. Woo J, Ho SC, Yuen YK, Yu LM, Lau J. An estimation of the functional disability burden in elderly Chinese age 70 years and over. Disabil Rehabil http://dx.doi.org/10.3109/09638289609166322.

3. Zeng Y, Wang ZL. "Gender Differences in Vitality of Oldest-old aged 80 years and over in China” Asian Development Bank policy consultancy report (internal paper); 2020. Variation No. 1 Contract No. 157665S27630.

4. Andersen-Ranberg K, Christensen K, Jeune B, Skytthe A, Vasegaard L, Vaupel JW. Declining physical abilities with age: a cross-sectional study of older twins and centenarians in Denmark. Age Ageing 1999;28 (4):373 - 7. http://dx.doi.org/10.1093/ageing/28.4.373.

5. Pi J, Olivé JM, Esteban M. Mini Mental State Examination: association of the score obtained with the age and degree of literacy in an aged population. Med Clin (Barc) 1994;103(17): 641-4. https://pubmed. ncbi.nlm.nih.gov/7808061/.

6. Blazer D, Burchett B, Service C, George LK. The association of age and depression among the elderly: an epidemiologic exploration. J Gerontol 1991;46(6):M210 - 5. http://dx.doi.org/10.1093/geronj/46.6.m210.

7. Van Oyen H, Nusselder W, Jagger C, Kolip P, Cambois E, Robine JM. Gender differences in healthy life years within the EU: an exploration of the "health-survival" paradox. Int J Public Health 2013;58(1):143 - 55. http://dx.doi.org/10.1007/s00038-012-0361-1.

8. Manton KG, Land KC. Active life expectancy estimates for the U.S. elderly population: a multidimensional continuous-mixture model of functional change applied to completed cohorts, 1982-1996. Demography 2000;37(3):253 - 65. http://dx.doi.org/10.2307/2648040. 


\section{SUPPLEMENTARY MATERIAL}

\section{Background Information}

The numbers and proportions of oldest-old aged 80 years and over who most likely need care in daily life and health service will increase much faster than the overall elderly populations in China and worldwide. In addition to the continued and significant decline of mortality rates especially at oldest-old ages, the main reason why the number of oldest-old will climb so quickly especially after 2030 is that China's baby boomers, those who were born in the 1950s and the 1960s, will become the "oldest-old" after 2030. Clearly, the oldest-old population is growing much faster than the overall elderly population.

\section{Data Source: The Chinese Longitudinal Healthy Longevity Study}

\section{(CLHLS), 1998-2018}

The first 8 waves of the Chinese Longitudinal Healthy Longevity Study (CLHLS) were conducted in 1998-2018. The 9th wave is being conducted in 2021 and has been expanded into the Chinese Longitudinal Healthy Longevity and Happy Family Study (CLHLS-HF) by adding several family-relevant questions based on CLHLS' initial questionnaire that contained about one-third of family-relevant questions. In this paper, we used the datasets of the CLHLS conducted in 1998-2018, which are summarized here.

The baseline survey of the CLHLS was conducted in 1998 and the follow-up surveys with new recruitments to replace dead participants were conducted in 2000, 2002, 2005, 2008-2009, 2011-2012, 2014, and 2017-2018. The CLHLS surveys were conducted in randomly selected roughly half of the counties and city districts of 23 out of 31 provincial level administrative divisions in China, including Liaoning, Jilin, Heilongjiang, Hebei, Beijing, Tianjin, Shanxi, Shaanxi, Shanghai, Jiangsu, Zhejiang, Anhui, Fujian, Jiangxi, Shandong, Henan, Hubei, Hunan, Guangdong, Guangxi, Sichuan, Chongqing, and Hainan. The survey areas covered 985,000,000 persons in the baseline year 1998 and 1,156,000 persons in the most recent census year 2010, about $85 \%$ of the total population of China.

In the 8 waves of the CLHLS conducted in 1998-2018, we have conducted over face-to-face home-based 113,000 interviews, including 19,500 centenarians, 26,800 nonagenarians, 29,700 octogenarians, 25,500 younger elders aged 65-79, and 11,300 middle-aged adults aged 35-64. Data on death dates/age and relatively detailed information of health status, care needs/costs, etc., before dying for the 28,900 elders aged 65-118 who died between waves were collected in interviews with a close family member of the deceased. All of the interviews, basic health exams, and DNA samples were voluntarily collected with standard consensus forms reviewed/signed by the participants (or their direct family members).

All centenarians who voluntarily agreed to participate in the study in the randomly selected roughly half of the counties and city districts of the 23 provincial level administrative divisions were interviewed. For each centenarian, 1 nearby nonagenarian (aged 90-99), 1 nearby octogenarian (aged 80-89), and 1.5 nearby young-old aged 65-79 were interviewed. "Nearby" was loosely defined — it could be in the same village or street if the interviewees of predesignated age and sex were available, or in the same town or in the same sampled county or city district if not available in the same village or street.

The pre-designated age and sex of the participants used to identify the approximately equal numbers of male and female nonagenarians, octogenarians, and young-old at each of the 5-year age groups from age 65 to 99 were randomly determined, based on the code numbers of the centenarians (1). The questionnaire data collected provides information on family structure, living arrangements and proximity to children, activities of daily living (ADL), the capacity of physical performance (picking up a book from the floor; standing up from a chair; and turning $360^{\circ}$ without help), self-reported health, self-evaluation of life satisfaction, cognitive functioning and mental health, chronic disease prevalence, care needs and costs, participation in social activities, diet, smoking and drinking behaviors, psychological characteristics, economic resources, and care giving and family support among elderly respondents and their relatives. Information about the health status of the CLHLS participants who were interviewed in the previous wave but died before the current survey was collected by interviewing a close family member. Information provided consists of cause of death, chronic diseases, ADL before dying, frequency of hospitalization or instances of being bedridden from the last interview until death, whether bedridden before death, 
length of disability, and suffering before death, etc.

The CLHLS has documented internationally established good data quality, including age reporting, assessments of mortality rates, proxy response, non-response rates, sample attrition, reliability and validity of major health measures, and rates of logically inconsistent answers (2-5). Relatively accurate age reporting of elderly including oldest-old among the Han Chinese ( $92 \%$ of total population of China) has been well-established and re-confirmed by a wide variety of international and domestic studies; this accuracy was due to the cultural traditions of memorizing one's exact date of birth to determine dates of important life events, such as dates of marriage and starting date to build a residential house $(6-6)$.

\section{Activities of daily living (ADL)}

\section{Measurements of the Health Statuses}

Self-reported six ADL, including eating, dressing, transferring, using the toilet, bathing, and continence, were useful measurements of functional capacity and service needs, as verified in numerous previous studies; ADL was also a significant predictor of mortality ( 7 ). Following the widely adopted practice in the literature, if none of the six $\mathrm{ADL}$ activities was impaired, the elder was classified as "ADL active"; otherwise, the elder was classified as "ADL impaired".

\section{Physical performance}

Self-reported ADL status may not always be accurate in measuring actual capacity in physical performance of the oldest-old, mainly because ADLs were also affected by household facilities (9). Also, some of the oldest-old may feel ashamed to report difficulties in daily activities such as continence. Therefore, as in other international healthy aging surveys, we conducted objective examinations to measure oldest-old participants' physical performance. We present and discuss in this paper the 1998, 2008, 2017-2018 waves of CLHLS results of gender differentials in the physical performance of standing up from a chair and picking up a book from the floor.

\section{The cognitive functional}

The cognitive functional statuses of the Chinese elderly were screened by the Chinese version of the Mini-Mental State Examination (MMSE), which were translated into the Chinese language based on the widely used international standard of the MMSE questionnaire with careful considerations of Chinese cultural and social context, and empirically tested in pilot survey interviews (9). We used the same cutoffs as the MMSE international standard, defining a score of $24+$ as "good" cognitive function and a score of $<24$ as impaired cognitive function (11-12).

\section{Self-reported health and life satisfaction}

Self-reported health and life satisfaction were significant and valuable predictors of the functioning and mortality of older adults, as demonstrated in many previous studies (13). In addition to self-reported health, the CLHLS also asked the elderly participants to report "How do you rate your life at the present?" The multiple responses to this question about life satisfaction were "very good; good; so-so; bad; very bad; and not able to answer." The category of self-reported "good life satisfaction" included those who provided an answer of "very good" or "good" to this question.

\section{REFERENCES}

1. Zeng Y. Towards Deeper Research and Better Policy for Healthy Aging --Using the Unique Data of Chinese Longitudinal Healthy Longevity Survey. China Economic J 2012;5(2-3):131-49. https://doi.org/10.1080/17538963.2013.764677.

2. Gu D. General Data Quality Assessment of the CLHLS. In: Yi Z, Poston DL, Vlosky DA, Gu D. (eds) Healthy Longevity in China. Demographic Methods and Population Analysis, vol 20. Springer, Dordrecht. https://doi.org/10.1007/978-1-4020-6752-5_3.

3. Zeng Y, Dudley L, Poston JR, Denese AV, Danan G. Healthy Longevity in China: Demographic, Socioeconomic, and Psychological Dimensions. Springer. Pp. xv_435. t134.95. ISBN: 978-1-4020-6751-8.

4. Chen H. Zeng Yi (ed.) Assessment of the quality of the cross-sectional data collected in the 2008-2009 wave of Chinese Longitudinal Healthy Longevity Survey. Research on Elderly Population, Family, Health and Care Needs/Costs 2010; Beijing: Science Press. (In Chinese).

5. Shen K. Assessment of the quality of the follow-up mortality data collected in the 2008-2009 wave of Chinese Longitudinal Healthy Longevity Survey. In: Zeng, Yi (ed.) Research on Elderly Population, Family, Health and Care Needs/Costs 2010; Beijing: Science Press. (In Chinese).

6. Coale AJ, Li SM. The effect of age misreporting in China on the calculation of mortality rates at very high ages. Demography 1991;28(2):293-301. https://doi.org/10.2307/2061281.

7. Wang Z, Zeng Y, Jeune B, Vaupel JW. Age Validation of Han Chinese Centenarians. GENUS - An International Journal of Demography Vol 1998, LIV: $123-41$.

8. Poston DL, Jr, Luo H. Age structure and composition of the Chinese minorities in 2000. Chinese Minority Populations 2004;19(3):9-15. (In Chinese). 
9. Scott WK, Macera CA, Cornman CB, Sharpe PA. Functional health status as a predictor of mortality in men and women over 65. J Clin Epidemiol 1997;50(3):291-6. doi: 10.1016/s0895-4356(96)00365-4.

10. Zeng, Y, Vaupel JW, Xiao Z, Zhang C, Liu Y. The Healthy Longevity Survey and the Active Life Expectancy of the Oldest Old in China. Population: An English Selection 2001;13(1):95-116. https://doi.org/10.2307/3030261

11. Deb S, Braganza J. Comparison of rating scales for the diagnosis of dementia in adults with Down's syndrome. J Intellect Disabil Res 1999;43 (Pt 5):400-7. https://doi.org/10.1046/j.1365-2788.1999.043005400.x.

12. Osterweil D, Mulford P, Syndulko K, Martin M. Cognitive function in old and very old residents of a residential facility: relationship to age, education, and dementia. J Am Geriatr Soc 1994;42(7):766-73. https://doi.org/10.1111/j.1532-5415.1994.tb06539.x.

13. Lee Y. The predictive value of self assessed general, physical, and mental health on functional decline and mortality in older adults. J Epidemiol Community Health. 2000;54(2):123-9. https://doi.org/10.1136/jech.54.2.123. 